\title{
Phenotypic Characterization and Molecular Mapping of Recessive Resistance To Meloidogyne Javanica in Cucumber, Cucumis Sativus
}

William Brock Rutter ( $\nabla$ william.rutter@usda.gov )

USDA Agricultural Research Service https://orcid.org/0000-0003-1748-2256

Abolfazl Hajihassani

University of Georgia

Yuhui Wang

Nanjing Agricultural University

Yiqun Wang

USDA-ARS Vegetable Crops Research Unit

Research Article

Keywords: Cucumber, Cucumis sativus, nematode resistance, Meloidogyne javanica, molecular markers, QTL mapping

Posted Date: October 22nd, 2021

DOI: https://doi.org/10.21203/rs.3.rs-919579/v1

License: (c) (i) This work is licensed under a Creative Commons Attribution 4.0 International License. Read Full License 


\section{Abstract}

The Javanese root-knot nematode (JRKN, Meloidogyne javanica) is a serious pest of cucumber (Cucumis sativus) grown in tropical and subtropical regions. The recessive $m j$ resistance gene first identified in the wild cucumber ( $C$. sativus var. hardwickii), is the only known source of resistance to JRKN within the $C$. sativus gene pool. Despite its potential utility as a nematode management tool, the $m j$ trait has yet to be fully characterized or widely incorporated into commercial cucumber lines. In this study, we mapped the $m$ j resistance locus to a 148 -kb interval on cucumber chromosome 1 by phenotyping a recombinant inbred line (RIL) and targeted backcross populations. The genomic interval is predicted to contain 36 candidate genes, including several known to be directly related to plant defense against pathogens. We also conducted histological comparisons of nematode development in mj resistant and susceptible lines, providing evidence that the mechanism of action for this gene may be different from that of known RKN resistance genes. These results provide a set of tightly linked markers that can assist breeding programs seeking to incorporate $m j$ resistance into new cucumber varieties. The candidate genes will also provide a starting point for further research into the mechanisms of action that underlay the mj-regulated JRKN resistance.

\section{Key Message}

A recessive host resistance gene against the Javanese root-knot nematode was mapped into a $148 \mathrm{~kb}$ region of cucumber chromosome 1 that is devoid of NB-LRR type R proteins.

\section{Introduction}

Root-knot nematodes (RKN, Meloidogyne spp.) are a serious yield-limiting pest of cucumber (Cucumis sativus L.). Two of the most damaging RKN species that infect cucumber are the southern root-knot nematode (SRKN, M. incognita), and the Javanese root-knot nematode (JRKN, M. javanica) (Hajihassani et al. 2019a). These nematodes invade host plant roots to form an elaborate feeding site and induce galling in the surrounding root tissue. Each female produces an egg mass containing hundreds of eggs that hatch and allow multiple cycles of reinfection throughout a growing season. The feeding and root galling caused by high populations of RKN disrupts normal root architecture, interferes with water and nutrient uptake, and reduces yields. Management of these pests in cucumber currently relies heavily on the use of expensive and environmentally damaging nematicides. Host genetic resistance is considered the most economical and environmentally friendly method for controlling RKNs in the field (Starr et al. 2002; Ayala-Doñas et al. 2020).

A number of resistance sources to RKNs have been identified in different crop plants, but the majority of these resistances have not been introgressed into elite crop cultivars (e.g., Williamson and Roberts 2009; Kaloshian and Teixeira 2019; Changkwian et al. 2019). In addition, only two have been cloned; the Mi-1.2 gene from tomato (Milligan et al., 1998; Vos et al. 1998) and the Ma gene from myrobalan plum (Claverie et al. 2011). Both genes encode canonical nucleotide binding leucine rich repeat (NLR) receptors that 
trigger a hypersensitive response against multiple species of RKN. Other dominant RKN resistance genes have been mapped to NLR rich regions in other crops (Davies and Elling 2015; Changkwian et al. 2019; Kaloshian and Teixeira 2019).

Limited work has been done in cucumber or other cucurbit crops on the genetic and molecular basis of RKN resistances. To date, the mj resistance gene is the only known source of RKN resistance in cucumber. Early screening tests identified this resistance to JRKN in the wild cucumber $C$. sativus var. hardwickii (HARD hereinafter), which was shown to be controlled by a single recessive gene, mj (Walters and Wehner 1997, 1998). Though $m$ j resistance is ineffective against SRKN, it is still a valuable trait that could help cucumber producers manage JRKN populations in the field. Despite its potential benefits for growers, $m j$ resistance has not yet been widely incorporated into commercially grown cucumber varieties likely due to its recessive inheritance combined with the time and labor necessary to phenotype roots for nematode resistance. Mapping the mj resistance trait within cucumber would reduce the need for time and labor-intensive nematode phenotyping by facilitating marker assisted selection.

In spite of its limitations, the mj resistance gene is unique in comparison to RKN resistance genes from other crops, which are single dominant in nature (e.g., Kaloshian and Teixeira 2019; Changkwian et al. 2019). The recessive nature of the $m j$ gene indicates that there may be different mechanisms confering JRKN resistance in cucumber. Knowledge of the unique molecular mechanisms underlying $m j$ resistance would facilitate a better understanding of the molecular basis of RKN pathogenesis in cucumber. In this study, we have further characterized the nature of the $m j$ resistance phenotype and map this locus into a 148-kb interval on cucumber chromosome 1. The genetic markers developed here will help breeders incorporate $m j$ resistance into new cucumber lines. This work also provides a starting point for pathologists seeking to investigate the molecular basis of RKN resistance.

\section{Materials And Methods}

\section{Plant materials and mapping populations}

Initial linkage mapping was performed with a recombinant inbred line $(R I L)$ population $(n=47)$ from the cross between Gy14 and WI7221 (PI 183967) which we used early for cucumber linkage map development and QTL analysis of horticulturally important traits (Ren et al. 2009; Sheng et al. 2020). Gy14 is an inbred line that is susceptible to JRKN while WI7221 was an inbred line selected from the wild cucumber HARD accession PI 183967 with high resistance to JRKN (Walters and Wehner 1997, 1998).

To narrow down the region for the mj locus, backcross populations were developed to identify recombinants defined by flanking markers from the initial linkage analysis. Since HARD is late flowering, a resistant RIL, RIL271 was used to cross with Gy14. RIL271 had normal flowering time similar to Gy14, and carried most Gy14 alleles at 995 SSR marker loci. First backcross $(B C 1)(n=190)$ plants were grown in 50-cell trays. DNA was extracted from each plant and screened with flanking markers to identify recombination events within the $m j$ interval. Individual plants $(n=14)$ found to have recombination 
events within the $m j$ interval were transplanted to 12" pots. Their genotypes were confirmed (see genotyping methods), and each was self-pollinated to produce $\mathrm{BC}_{1} \mathrm{~S}_{1}$ seed. We selected true breeding $\mathrm{S} 1$ plants, homozygous for the $m j$ introgression, which were again confirmed by genotyping and selfpollinated to produce the BC1S2 plants used for phenotyping for $m j$ resistance. Each line was phenotyped with at least 2 replicates in independent screens.

\section{Histological analysis of nematode development in roots}

Histological examinations were performed to investigate the development of JRKN within the susceptible Gy14 and resistant RIL271 lines as previously described (Hajihassani et al. 2020). Briefly, three galled root segments were randomly collected from each infected genotype. The galled roots were fixed in a formaldehyde solution for 1-2 days at room temperature. The fixed roots were dehydrated in a series of ethanol solution (70 to 100\%) using a tissue processor (Excelsior AS; Thermo Scientific, Thermo Shandon Limited, Runcorn, Cheshire, U.K.) and embedded in paraffin. Cross-sections (4 to $5 \mu \mathrm{m}$ thick) were prepared using a rotary microtome (RM2125 RTS; Leica, Leica Biosystems, Nussloch, Germany) and transferred to glass slides. Cross-sections were stained in hematoxylin-eosin, examined under a microscope (Olympus BX43; Olympus, Tokyo, Japan), and photographed using a digital camera (Olympus DP73; Olympus).

Root penetration assays were conducted to compare the number of juvenile nematodes entering the root tips of susceptible and resistant control lines. Plants from each line were individually planted in D40L Deepot@ containers (Stuewe \&Sons, Tangent Oregon) filled with an autoclave sterilized sand:soil mixture and arranged in a randomized complete block design. Each plant was inoculated with 300 infective stage 2 juvenile (J2) JRKN. Root systems of 4 inoculated plants from each line were collected at 2, 4, and 6 days post-inoculation (DPI). At each time point, root systems were washed to remove soil, soaked in a $1.5 \% \mathrm{NaOCl}$ solution for $3 \mathrm{~min}$, and rinsed with tap water. The J2 nematodes within the roots were stained by boiling for $30 \mathrm{sec}$ in red food coloring (Thies et al. 2002). Roots were destained by soaking in lactophenol for 48 hours, then pressed between two glass slides as previously described (Hajihassani et al. 2019b). The number of stained J2 within each root tip was counted under a stereomicroscope. J2 counts were recorded and tested for significant differences using the 2-way student t-test in Excel.

\section{Screening for JRKN resistance}

Phenotyping of 47 RILs and 14 recombinant backcross lines was performed in a greenhouse in eight independent screening tests. Each RIL was phenotyped at least 3 times across independent screens. In each experiment, seeds from each line were germinated under moist filter paper for 24 hours at $28^{\circ} \mathrm{C}$ before being planted directly into D40L Deepot ${ }^{\mathrm{TM}}$ containers (Stuewe \&Sons, Tangent Oregon) filled with an autoclave sterilized 1:1 sand:potting soil mix. Plants were arranged into a randomized block design, watered and fertilized using a soluble 20-20-20 fertilizer as necessary throughout the duration of each screen. Each block included the susceptible control line (Gy14) and a resistant control line (HARD or RIL271). 
Two weeks after planting, each plant was inoculated with 3,000 JRKN eggs. The JRKN isolate 'VW4', originally isolated by Professor Valerie Williamson at the University of California, was used as inoculum for all the screens in this study. The nematode culture was maintained in the greenhouse on susceptible 'Rutgers' tomato grown in autoclave sterilized media. On the day of inoculation, nematode eggs were extracted from galled tomato roots by agitating them in a $0.5 \% \mathrm{NaOCl}$ solution for 2 minutes, and rinsing the mixture through stacked 8" sieves ( No. 270 over No. 500) as previously described (Hussey and Barker 1973). The eggs were counted under a dissecting microscope, diluted in tap water, and $4 \mathrm{~mL}$ of a 750 $\mathrm{egg} / \mathrm{mL}$ mixture was pipetted into the soil at the base of each plant.

Five weeks post-inoculation, cucumber roots were washed free of soil and stained for the presence of egg masses using red food coloring as previously described (Thies et al. 2002). The total number of visible egg masses was counted for each root system. The distinction between susceptible and resistant lines was determined by the average number of egg masses on susceptible and resistant controls. Cucumber lines averaging less than 15 nematode egg masses were classified as resistant and lines averaging more than 30 nematode egg masses per root system were classified as susceptible.

\section{Linkage/QTL analysis}

The JRKN resistance in the $47 \mathrm{RIL}$ lines was treated as either a qualitative or quantitative trait. Linkage analysis was performed with JoinMap 3.0 and QTL analysis was performed with the mean egg mass per root system for each RIL using Composite Interval Mapping (CIM) approach as previously described (Wang et al. 2016). Genotypic data for these 47 RILs were from a linkage map developed previously that contains 995 SSR marker loci (Ren et al. 2009). The analysis was conducted using R/qtl (Broman et al. 2003). Significance of the $m j$ resistance QTL was determined using a 1,000 permutation test, and an initial 1.5-LOD support interval was used to identify the flanking SSR markers (SSR00160 and SSR22110) on either side of the initial $1.23 \mathrm{Mb}$ interval. These flanking markers were used to screen BC1 populations and identify 14 independent recombination events that were used to further narrow down the $m j$ interval.

\section{Marker development and analysis}

To refine the map locations of the mj locus, SNPs (single nucleotide polymorphisms) were identified by aligning genomic DNA sequences in the target region between the two parental lines Gy14 (v2.0 chr1: 4,031,065 - 5,261,376) and PI 183967 (Chr1:4,043,287-5,270,956), which were available on the Cucurbit Genetics Database (CuGenDB, http://cucurbitgenomics.org/). Primer sequence information for all SNP markers developed in this study is provided in Supplemental Table S1. DNA sequences harboring SNPs were sent to C3r Bioscience (Essex, UK) for PCR Allele Competitive Extension (PACE ${ }^{\mathrm{TM}}$ ) assay design, or LGC Biosearch Technologies (Middlesex, UK) for Kompetitive Allele Specific PCR (KASP ${ }^{\mathrm{TM}}$ ) assay design (Table S1). The primers for each PACE assay were synthesized by Integrated DNA Technologies (Research Triangle Park, NC) and were reconstituted and mixed according to the manufacturer's instructions.

Total DNA used for genotyping was extracted from leaf samples using the Plant DNAeasy Mini kit (Qiagen, Hilden, Germany) according to the manufacturer's protocols. PACE and KASP genotyping assays 
were run in $5 \mu \mathrm{l}$ reactions in 96-well plate format on a Light Cycler $480^{\mathrm{TM}}$ (Roche Diagnostics Corporation, Indianapolis, IN). Each reaction contained $1.5 \mu \mathrm{l}$ of DNA template [10ng/uL] along with $2.5 \mu \mathrm{l} 2 \mathrm{x} \mathrm{KASP}$ Master Mix (LGC Biosearch Technologies, Middlesex, UK), $0.07 \mu \mathrm{l}$ reconstituted PACE or KASP assay, and $0.93 \mu \mathrm{lddH_{2 }}$ O. The thermal cycler protocol consisted of (in the sequence presented): $94^{\circ} \mathrm{C}-15 \mathrm{~min}, 10$ cycles of $94^{\circ} \mathrm{C}-20 \mathrm{sec}$ and $61^{\circ} \mathrm{C}\left[-0.6^{\circ} \mathrm{C} / \mathrm{cycle}\right]-1 \mathrm{~min}, 26$ cycles of $94^{\circ} \mathrm{C}-20 \mathrm{sec}$ and $55^{\circ} \mathrm{C}-1 \mathrm{~min}$, and cooled to $37^{\circ} \mathrm{C}$ for fluorescent signal quantification in the HEX (533-580nM) and FAM (465-510nM) wavelengths. Raw signals for each well were exported for analysis using custom scripts in R. All genotyping plates contained at least two no template control reactions as well as 2 reactions containing DNA from each parental line (Gy14 and RIL271) to facilitate visual end-point genotype clustering.

Each PCR reaction for SSR markers contained 50ng of template DNA, $0.5 \mu M$ of each primer, $1 \mathrm{U}$ Taq DNA polymerase (Thermo Fisher Scientific), $2.5 \mu \mathrm{l} 10 \times \mathrm{PCR}$ buffer, $1.5 \mathrm{mM} \mathrm{MgCl}_{2}$, and $0.2 \mathrm{mM}$ dNTPs in a total volume of $25 \mu \mathrm{l}$. A one-fit-all, touch-down PCR program was used for all SSR primer sets and consisted of (in the sequence presented): $3-\mathrm{min}$ at $95^{\circ} \mathrm{C}$; six cycles of $45 \mathrm{sec}$ at $94^{\circ} \mathrm{C}, 5 \mathrm{~min}$ at $68^{\circ} \mathrm{C}, 1 \mathrm{~min}$ at $72^{\circ} \mathrm{C}$, with the annealing temperature being reduced by $2^{\circ} \mathrm{C}$ per cycle; eight cycles of $45 \mathrm{sec}$ at $94^{\circ} \mathrm{C}, 2$ min at $58^{\circ} \mathrm{C}, 1 \mathrm{~min}$ at $72^{\circ} \mathrm{C}$, with the annealing temperature reduced by $1^{\circ} \mathrm{C}$ per cycle; a final 25 cycles of $45 \mathrm{sec}$ at $94^{\circ} \mathrm{C}, 2 \mathrm{~min}$ at $50^{\circ} \mathrm{C}$ and $1 \mathrm{~min}$ at $72^{\circ} \mathrm{C}$. PCR products were separated and imaged in a $4 \%$ MetaPhor $^{\text {TM }}$ (Lonza, Rockland ME) agarose gel.

\section{Results}

\section{Resistance conferred by mj inhibits development and reproduction of JRKN in the roots}

Cucumber roots carrying the $m$ j resistance gene produced significantly fewer nematode egg masses compared to susceptible controls. The resistant lines HARD and RIL271 averaged just 2.8 egg masses per root system across 25 phenotyped plants, and we never observed more than 8 egg masses on any of these root systems. Within the same screens, the susceptible parental line, Gy14, averaged 66.1 egg masses per root system, and individual root systems had between 15 and 185 egg masses (Fig. 1a and 2a). Gy14 displayed a significantly higher number of egg masses per root system than HARD or RIL271 $(p<0.05)$ across all phenotyping screens.

Though there were nematode induced galls on both resistant and susceptible lines (Fig. 1b and 1c), we observed drastic differences in the development of the nematode and its feeding site within galls produced on resistant RIL271 compared to those produced on susceptible Gy14. Well-developed nematodes and giant cell feeding sites were observed in histological sections made from galls produced on Gy14 (Fig. 1d). Conversely, no feeding sites were observed in any of the gall sections collected from RIL271 (Fig. 1e). And although some nematodes were observed in the sections made from RIL271 galls, these nematodes were less well developed than those found in the sections from Gy14 galls.

Despite these differences between nematode development in the galls of resistant and susceptible lines, we observed no significant differences in the number of $\mathrm{J} 2$ nematodes that penetrated the roots of 
RIL271 versus Gy14 at 2, 4, or 6 DPI (supplemental Table S2). These observations indicated that $m$ jconferred resistance inhibits JRKN development later in the infection process, preventing the nematodes from developing and producing egg masses.

\section{JRKN resistance in HARD is controlled by a single recessive gene on $\mathrm{Chr} 1$}

We recorded number of galls and egg masses produced on 47 RILs, Gy14, HARD and their F1 in multiple screening tests. The percentages of galls, mean egg masses, and their range for each test line are presented in supplemental Table S3, and the distribution of mean egg masses among RILs is graphically presented in Fig. 2a. The HARD, Gy14 and their F1 had 2.3, 66.1, and 59.8 egg masses, with corresponding $29.0 \%, 64.8 \%$, and $67.6 \%$ galling rate, respectively. Both parameters also exhibited significant differences among RILs. Using mean egg mass of 10.0 as the cutoff, 21 and 26 RILs were classified as resistant and susceptible, respectively, which was consistent with expected 1:1 segregation ratio ( $P=0.4658$ in Chi-square test). These observations support previous work suggesting JRKN resistance in PI 183967 was controlled by a single recessive gene, mj (Walters and Wehner 1998).

QTL analysis with mean egg mass of the 47 RILs identified a single peak with high LOD support (> 20) on cucumber chromosome 1 (Chr1, Fig. 2b) which was consistent with phenotypic analysis for JRKN resistance. This major-effect QTL explains $90 \%$ of observed phenotypic variance (PVE or $R^{2}$ ), and the 1.5 LOD drop interval of this QTL was defined by two flanking markers SSR00160 and SSR22110, which were $1.23 \mathrm{Mbp}$ apart on Chr1 (Gy14v2.0). We also conducted linkage analysis using qualitative data of the RILs (resistant vs susceptible, Table S3). The resulting linkage map is shown in Fig. 2c. The mj locus was mapped on Chr1 flanked by the same markers as in QTL analysis.

\section{Refinement of mj map location}

To further narrow down the candidate region for the $m j$ locus, we developed backcross populations from the cross between Gy14 and the resistant line RIL271 using Gy14 as the recurrent parent. We screened 190 BC1 individuals with two flanking KASP markers (KASP_GY1G006250 and KASP_GY1G008330) and selected $14 \mathrm{BC} 1$ plants with recombination between the two markers $(1.23 \mathrm{Mb})$. JRKN resistance screening of these lines revealed that 8 were resistant and 6 were susceptible (supplemental Table S3). New KASP and PACE markers were developed in this region (Table S1) which were used to genotype these recombinants, allowing us to narrow down the candidate mj region to a 148-kb interval in the Gy14 v2.0 genome (Fig. 2d). Thirty-six genes were annotated in this region which are listed in supplemental Table S4. Among them, a number of genes had predicted functions related to plant defense including 10 genes predicted to be receptor-like kinases, 4 of which were further annotated as 'Leaf Rust 10 Disease Resistance Locus' and a 5th gene annotated as "PR5-like receptor kinase". Another notable candidate gene (CsGy1G006630) was annotated as 'SAR Deficient 1' (Table S4).

\section{Discussion}


Our molecular mapping work in indicates that the $m$ j resistance gene is located in a 148-kb region of cucumber Chr 1 with 36 predicted genes. Among the candidates are 10 genes annotated as receptor-like protein kinase (RLK) resistance proteins including 4 'Leaf Rust 10 Disease-Resistance' proteins within the Gy14v2.0 genome. RLKs are the main cell surface receptors that initiate PAMP Triggered Immunity (PTI) in plants, and often respond to broadly conserved pathogen signatures such as flagellin or chitin (Jones and Dangl 2006). RLKs that respond broadly to plant parasitic nematodes have been identified in Arabidopsis (Mendy et al. 2017). Though the majority of RLK-controlled resistance phenotypes are dominantly inherited, a RLK gene in cucumber has been found to confer recessively inherited resistance to the downy mildew pathogen (Berg et al. 2020). There are several other candidate genes within the $m j$ interval with predicted functions related to plant defense, including a 'SAR Deficient 1'(CsGy1G006630) and several other predicted transcription factor genes from protein families that are known to have roles in plant-nematode interactions. For example, several MYB transcription factors (similar to candidate CsGy1G006530) have been shown to have roles in cyst nematode feeding-site development (Piya et al. 2017; Wiśniewska et al. 2021).

The recessive nature of the $m j$ gene indicates that it is more likely to be a loss-of-susceptibility mechanism, similar to those seen in other recessive pathogen resistance genes in cucumber such as STAYGREEN and CSMLO1 genes (Nie et al. 2015; Wang et al. 2019). These two loss-of-susceptibility genes have been successfully used to manage other cucumber pathogens in the field, and their unique mechanisms of action are thought to make them more durable than other canonical NLR genes ( $\mathrm{Li}$ et al. 2001). Novel loss-of-susceptibility mechanisms have also been reported for recessive resistance genes in other plant-pathosystems (Büschges et al. 1997; Deslandes et al. 2002; lyer and McCouch 2004; Yuan et al. 2009). Though further work is needed to identify and characterize the specific mj resistance gene, none of the 36 candidate genes are NLR genes, indicating that the mj gene is a unique RKN resistance gene.

The resistance phenotype of the mj gene is also unique compared to other known RKN resistance genes. Though JRKN can induce galls on resistant mj genotypes, its ability to reproduce is almost completely suppressed. Our histological comparisons of galls produced on resistant and susceptible genotypes indicate that the nematode and its feeding site fail to develop within the roots of resistant lines. Similar histological investigations into RKN resistance genes in other RKN-pathosystems have been reported to have effects very early on in infection; often reducing the numbers of $\mathrm{J} 2$ within the roots early in infection well before gall development (Pegard et al. 2005; Williamson and Kumar 2006; Hajihassani et al. 2019b). The development of galls on $\mathrm{mj}$ resistant roots and the similar numbers of $\mathrm{J} 2$ nematodes observed penetrating susceptible and resistant roots up to 6 days post-inoculation suggests that $m j$ resistance takes effect later in nematode development compared to other RKN resistance genes. Further dissection of the molecular mechanisms involved in this unique $\mathrm{mj}$ resistance could provide valuable insight into cucumber resistance against RKN.

This study also provides plant breeders with a set of molecular markers (Table S1) that could be used to incorporate the mj locus into new horticulturally useful lines. This is particularly important in the case of 
cucumber, which is highly susceptible to damage by RKN and has few known sources of resistance to any Meloidogyne species. Genetic mapping of the mj locus from this study provides a starting point for pathologists seeking to further investigate $m j$ resistance mechanisms. To our knowledge, the $m j$ locus is still the only characterized source of RKN resistance within the genus Cucumis.

\section{Declarations}

\section{Acknowledgments}

The Authors would like to thank Sharon Buckner and Jennifer MacMillan for their technical assistance in conducting the phenotyping screens for this manuscript. They would also like to thank Professor Valerie Williamson for generously sharing her Meloidogyne javanica isolate "VW4".

Funding: Work in YQW's lab was supported by the Agriculture and Food Research Initiative Competitive Grants under Award Numbers 2015-51181-24285 and 2017-67013-26195 from the US Department of Agriculture National Institute of Food and Agriculture (to Y.Weng). USDA is an equal opportunity provider and employer.

\section{Author contributions}

WR conducted nematode resistance screening, developed and screened BC populations, and supervised work in WR's lab. AH conducted all histological analysis of susceptible and resistant lines, and supervised work in AH's lab. YQW conceived the experiment, developed the RIL population, and supervise work in YW's Lab. YQW also participate in writing and revising the manuscript. YHW conducted QTL analysis of the data.

\section{Availability of data and material}

All raw data will be made available upon request

\section{Conflict of interest statement}

The authors declare no conflict of interest.

\section{Code availability}

Not applicable

\section{Ethics approval}

Not applicable

\section{Consent to participate}

Not applicable 


\section{Consent for publication}

All authors have reviewed and consent to publication

\section{References}

1. Ayala-Doñas A, Cara-García M de, Talavera-Rubia M, Verdejo-Lucas S (2020) Management of soilborne fungi and root-knot nematodes in cucurbits through breeding for resistance and grafting. Agronomy 10:1641. https://doi.org/10.3390/agronomy10111641

2. Berg JA, Hermans FWK, Beenders F et al (2020) Analysis of QTL DM4.1 for downy mildew resistance in cucumber reveals multiple subqtl: a novel rlk as candidate gene for the most important subQTL. Front Plant Sci 11:569876. https://doi.org/10.3389/fpls.2020.569876

3. Broman KW, Wu H, Sen S, Churchill GA (2003) R/qtl: QTL mapping in experimental crosses. Bioinformatics 19:889-890. https://doi.org/10.1093/bioinformatics/btg112

4. Büschges R, Hollricher K, Panstruga R et al (1997) The barley mlo gene: a novel control element of plant pathogen resistance. Cell 88:695-705. https://doi.org/10.1016/S0092-8674(00)81912-1

5. Changkwian A, Venkatesh J, Lee J-H et al (2019) Physical localization of the root-knot nematode (Meloidogyne incognita) resistance locus Me7 in pepper (Capsicum annuum). Front Plant Sci 10:886. https://doi.org/10.3389/fpls.2019.00886

6. Claverie $\mathrm{M}$, Dirlewanger $\mathrm{E}$, Bosselut $\mathrm{N}$ et al (2011) The Ma gene for complete-spectrum resistance to Meloidogyne species in Prunus Is a TNL with a huge repeated C-terminal post-LRR region. Plant Physiol 156:779-792. https://doi.org/10.1104/pp.111.176230

7. Davies LJ, Elling AA (2015) Resistance genes against plant-parasitic nematodes: a durable control strategy? Nematology 17:249-263. https://doi.org/10.1163/15685411-00002877

8. Deslandes L, Olivier J, Theulieres F et al (2002) Resistance to Ralstonia solanacearum in Arabidopsis thaliana is conferred by the recessive rrs 1-rgene, a member of a novel family of resistance genes. Proc Natl Acad Sci 99:2404-2409. https://doi.org/10.1073/pnas.032485099

9. Hajihassani A, Davis RF, Timper P (2019a) Evaluation of selected nonfumigant nematicides on increasing inoculation densities of Meloidogyne incognita on cucumber. Plant Dis 103:3161-3165. https://doi.org/10.1094/PDIS-04-19-0836-RE

10. Hajihassani A, Rutter WB, Luo X (2019b) Resistant pepper carrying N, Me1, and Me3 have different effects on penetration and reproduction of four major Meloidogyne species. J Nematol 51:1-9. https://doi.org/10.21307/jofnem-2019-020

11. Hajihassani A, Rutter WB, Schwarz T et al (2020) Characterization of resistance to major tropical root-knot nematodes (Meloidogyne spp.) in Solanum sisymbriifolium. Phytopathology 110:666-673. https://doi.org/10.1094/PHYTO-10-19-0393-R

12. Hussey RS, Barker KR (1973) Comparison of methods of collecting inocula of Meloidogyne-Spp. Including a New Technique. Plant Dis Report 57:1025-1028 
13. Iyer AS, McCouch SR (2004) The rice bacterial blight resistance gene $x a 5$ encodes a novel form of disease resistance. Mol Plant-Microbe Interactions 17:1348-1354. https://doi.org/10.1094/MPMI.2004.17.12.1348

14. Jones JDG, Dangl JL (2006) The plant immune system. Nature 444:323-329. https://doi.org/10.1038/nature05286

15. Kaloshian I, Teixeira M (2019) Advances in plant - nematode interactions with emphasis on the notorious nematode genus Meloidogyne. Phytopathology 109:1988-1996. https://doi.org/10.1094/PHYTO-05-19-0163-IA

16. Li Z-K, Sanchez A, Angeles E et al (2001) Are the dominant and recessive plant disease resistance genes similar?: A case study of rice $\mathrm{R}$ genes and Xanthomonas oryzae pv. oryzae races. Genetics 159:757-765. https://doi.org/10.1093/genetics/159.2.757

17. Mendy B, Wang'ombe MW, Radakovic ZS et al (2017) Arabidopsis leucine-rich repeat receptor-like kinase NILR1 is required for induction of innate immunity to parasitic nematodes. PLOS Pathog 13:e1006284. https://doi.org/10.1371/journal.ppat.1006284

18. Milligan SB, Bodeau J, Yaghoobi J et al (1998) The root knot nematode resistance gene Mi from tomato is a member of the leucine zipper, nucleotide binding, leucine-rich repeat family of plant genes. Plant Cell 10:1307-1319. https://doi.org/10.1105/tpc.10.8.1307

19. Nie J, Wang Y, He H et al (2015) Loss-of-function mutations in csmlo1 confer durable powdery mildew resistance in cucumber (Cucumis sativus L.). Front Plant Sci 6:1155. https://doi.org/10.3389/fpls.2015.01155

20. Pegard A, Brizzard G, Fazari A et al (2005) Histological characterization of resistance to different root-knot nematode species related to phenolics accumulation in Capsicum annuum. Phytopathology 95:158-165. https://doi.org/10.1094/Phyto-95-0158

21. Piya S, Kihm C, Rice JH et al (2017) Cooperative regulatory functions of miR858 and MYB83 during cyst nematode parasitism. Plant Physiol 174:1897-1912. https://doi.org/10.1104/pp.17.00273

22. Ren Y, Zhang Z, Liu J et al (2009) An integrated genetic and cytogenetic map of the cucumber genome. PloS One 4:e5795. https://doi.org/10.1371/journal.pone.0005795

23. Sheng Y, Pan Y, Li Y et al (2020) Quantitative trait loci for fruit size and flowering time-related traits under domestication and diversifying selection in cucumber (Cucumis sativus). Plant Breed 139:176-191. https://doi.org/10.1111/pbr.12754

24. Starr J, Bridge J, Cook R (2002) Resistance to plant-parasitic nematodes: history, current use and future potential. Plant Resist Parasit Nematodes 1-22. https://doi.org/10.1079/9780851994666.0001

25. Thies JA, Merrill SB, Corley EL (2002) Red food coloring stain: new, safer procedures for staining nematodes in roots and egg masses on root surfaces. J Nematol 34:179-181

26. Walters SA, Wehner TC (1997) "Lucia”, "Manteo", and "Shelby" root-knot nematode-resistant cucumber inbred lines. Hortscience 32:1301-1303. https://doi.org/10.21273/HORTSCI.32.7.1301 
27. Walters SA, Wehner TC (1998) Independence of the $m j$ nematode resistance gene from 17 gene loci in cucumber. Hortscience 33:1050-1052. https://doi.org/10.21273/HORTSCI.33.6.1050

28. Wang Y, Tan J, Wu Z et al (2019) Staygreen, stay healthy: a loss-of-susceptibility mutation in the STAYGREEN gene provides durable, broad-spectrum disease resistances for over 50 years of US cucumber production. New Phytol 221:415-430. https://doi.org/10.1111/nph.15353

29. Wang $Y$, VandenLangenberg $K$, Wehner TC et al (2016) QTL mapping for downy mildew resistance in cucumber inbred line WI7120 (PI 330628). Theor Appl Genet 129:1493-1505. https://doi.org/10.1007/s00122-016-2719-x

30. Williamson V, Kumar A (2006) Nematode resistance in plants: the battle underground. Trends Genet 22:396-403. https://doi.org/10.1016/j.tig.2006.05.003

31. Wiśniewska A, Wojszko K, Różańska E et al (2021) Arabidopsis thaliana Myb59 gene is involved in the response to Heterodera schachtii infestation, and its overexpression disturbs regular development of nematode-induced syncytia. Int J Mol Sci 22:6450. https://doi.org/10.3390/ijms22126450

32. Yuan M, Chu Z, Li X et al (2009) Pathogen-induced expressional loss of function is the key factor in race-specific bacterial resistance conferred by a recessive $\mathrm{R}$ gene $x a 13$ in rice. Plant Cell Physiol 50:947-955. https://doi.org/10.1093/pcp/pcp046

\section{Figures}



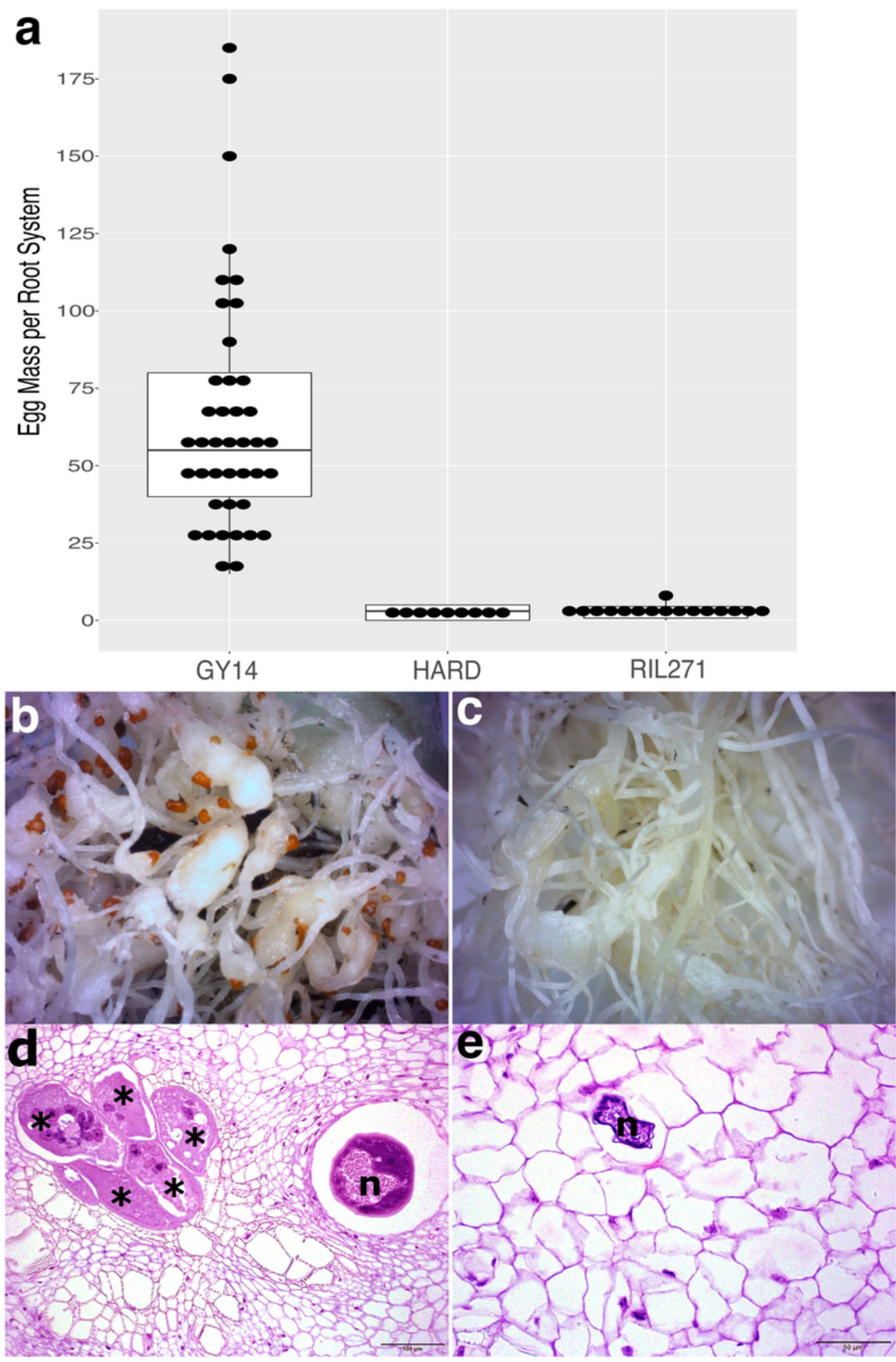

\section{Figure 1}

A comparison of resistant and susceptible cucumber roots five weeks after infection with JRKN. a.) The susceptible parental line (Gy14) showed significantly more nematode egg masses than either resistant control lines HARD or RIL271. b) Numerous nematode galls with egg masses (stained red) developed on the roots of Gy14. c) Though some galls did develop on resistant RIL271 in response to nematode infection, egg masses did not. d) Histological sectioning and staining of galls produced on Gy14 revealed 
well developed giant cell feeding sites $\left(^{*}\right)$ and nematodes $(n)$ that were clearly visible. e) Sections made from the galls produced on RIL271 had no obvious feeding sites and relatively small nematodes (n).

\section{Figure 2}

Phenotyping and QTL mapping results from a population of 47 RILs and 14 recombinant BC1 lines derived from the cross between Gy14 and PI 183967 (HARD). a) Histogram of the mean number of JRKN egg masses per root system collected from 8 independent phenotyping screens, including the resistant parent (HARD, red), the susceptible parent (Gy14, green), and F1 individuals (green). The phenotype distribution was consistent with a single recessive gene. b) QTL analysis using the RIL population data identified a single $1.23 \mathrm{Mb}$ region on chromosome 1 for the mj locus (LOD > 20). c) Its map location is consistent with result from linkage analysis with JRKN resistance as a qualitative trait. d) The physical map depicts the relative locations of markers in relation to the 148-kb mj interval, resolved using the 14 recombinant BC1 lines.

\section{Supplementary Files}

This is a list of supplementary files associated with this preprint. Click to download.

- SupplementalTablesS1S491721.xlsx 